\title{
The Correlation between E-Selectin S128R Gene Poly- morphism and Ischemic Stroke in Chinese Population : A Meta-Analysis
}

\author{
Xitong Yang, Rong Ma, Yuanyuan Zhang, Guangming Wang \\ Genetic Testing Center, The First Affiliated Hospital of Dali University, Dali, China
}

To perform a systematic review of the data collected from case-control studies conducted earlier to investigate the correlation between E-selectin S128R polymorphism and ischemic stroke (IS) risk among the Chinese population. The PubMed, Web of Science, Chinese biomedical literature database (CBM), Chinese databases China National Knowledge Infrastructure (CNKI), WanfangData knowledge service platform (Wanfang Data), and information resource integration service platform (VIP) Databases were searched to retrieve case-control studies on the correlation between E-selectin gene S128R polymorphism and IS from the inception of the database till June 2019. The literature was screened, data were extracted, the risk of bias was reviewed, and the studies included were assessed independently by two reviewers. Stata ver. 12.0 software (Stata Corp LLC, College Station, TX, USA) was used to perform the meta-analysis. A total of 2907 cases from eight case-control studies involving $1478 \mathrm{IS}$ patients and 1429 controls were included in this study. The R allele and RS genotype in E-selectin were found to be associated with the risk of IS as per the results of the meta-analysis (R vs. S : odds ratio [OR], $2.75 ; 95 \%$ confidence interval $[\mathrm{Cl}], 2.15-3.51 ; p<0.00001 ; \mathrm{RS}$ vs. SS : $\mathrm{OR}, 2.50 ; 95 \% \mathrm{Cl}, 1.95-3.19 ; p<0.00001$; $\mathrm{RR}+\mathrm{RS}$ vs. SS : $\mathrm{OR}, 2.85,95 \% \mathrm{Cl}, 2.21-3.67 ; p<0.00001)$. The E-selectin gene S128R polymorphism is likely related to IS based on the results of a meta-analysis in the Chinese population, and the R allele and RS genotype of E-selectin may be IS risk factors.

Key Words : E-selectin · Polymorphism · Stroke · Meta-analysis.

\section{INTRODUCTION}

Ischemic stroke (IS) is one of the most complex cerebral diseases, with high morbidity, disability, recurrence, and death rate. Each year, about 15 million individuals suffer from stroke across the world, of which $20 \%$ are fatal. More than $50 \%$ of sufferers survive but remain dependent on others for daily activities $^{6,18}$. Each year, about 5.7 million people die from stroke, and $87 \%$ of the patients are from low- or middle-income countries. IS significantly affects people's quality of life and also gives rise to a series of complications, which cause pain to the patients and increase the burden of the family and society ${ }^{4)}$. IS is the second highest cause of death in China and the third highest in the world and is one of the main causes of adult disability. IS is a nervous system disease influenced by multiple complex factors including both environmental and

- Received : November 1, 2019 •Revised : December 6, 2019 •Accepted : December 17, 2019

- Address for reprints : Guangming Wang

Genetic Testing Center, The First Affiliated Hospital of Dali University, 32 Jiashibo Road, Dali, Yunnan 671000, China

Tel : +86-872-2201096, Fax : +86-872-2201096, E-mail : wgm1991@dali.edu.cn, ORCID : https://orcid.org/0000-0002-0220-1493

This is an Open Access article distributed under the terms of the Creative Commons Attribution Non-Commercial License (http://creativecommons.org/licenses/by-nc/4.0) which permits unrestricted non-commercial use, distribution, and reproduction in any medium, provided the original work is properly cited. 
genetic factors. About $80 \%$ of IS cases are reported to be related closely to genetic factors, as certified by epidemiological evidence $^{5)}$.

E-selectin is an adhesion molecule and expressed only on endothelial cells after activation by cytokines such as interleukin 1 , tumor necrosis factor $\alpha$ or bacterial lipopolysaccharides $^{10)}$. Several polymorphisms in the E-selectin gene have been reported and it was recently noted that S128R E-selectin gene polymorphism is hardly investigated in relation to stroke. E-selection is a kind of surface glycoprotein, an important endothelial adhesion factor that regulates leukocyte binding and blood infiltration to inflammatory sites. The E-selectin gene is composed of 14 exons and 13 introns, a variation of an adenine by cytosine at the 561 gene position causes a serine to arginine (S128R) change in the protein. Das et al. ${ }^{2)}$ and Roy et al. ${ }^{9)}$ in their research found that E-selectin S128R gene polymorphism was related to IS, but ethnic and regional differences led to uncertainty of the findings. Thus, in this study, we performed a meta-analysis combining results from published articles on the Chinese population to investigate the association between E-selectin gene polymorphism and IS among the Chinese population to obtain a more precise and comprehensive estimate.

\section{METHODS}

\section{Search strategy}

The databases of PubMed, Web of Science, Chinese biomedical literature database (CBM), Chinese databases China National Knowledge Infrastructure (CNKI), WanfangData knowledge service platform (Wanfang Data), and information resource integration service platform (VIP) were searched by two authors (X.T. and R.M.) independently; the deadline for publication was June 2019. The searched terms were "ischemic OR stroke OR cerebrovascular disease OR brain hemorrhage OR intracranial hemorrhage OR cerebral hemorrhage OR cerebral infarction" and "E-selectin S128R" OR "E-selectin A561C" and "polymorphism OR SNP." Review articles and reference lists on the basis of these terms were obtained manually to find additional relevant studies.

\section{Data extraction}

The two investigators (X.T. and H.Y.) individually extracted essential information from the eligible articles. The following necessary data and information were extracted : 1) participant characteristics including mean age, gender, and country of origin and the total numbers of cases and controls; 2) the basic characteristics of the study (including the title, published year, author's names, race, etc.); and 3) cases and controls for each genotype characteristic and genotype distribution were in line with the Hardy-Weinberg equilibrium (HWE).

\section{Inclusion and exclusion criteria}

Inclusion criteria : studies 1) that evaluated the relationship between E-selectin S128R (A561C) polymorphisms and IS risk; 2) which had a case-control design; 3) or interventions that included IS patients in the case group and healthy individuals in the control group; 4) in which data analysis included the odds ratio (OR), risk ratio, and the corresponding 95\% confidence interval (CI); 5) in which the genotype distribution in the control group was in line with the HWE; and 6) con-

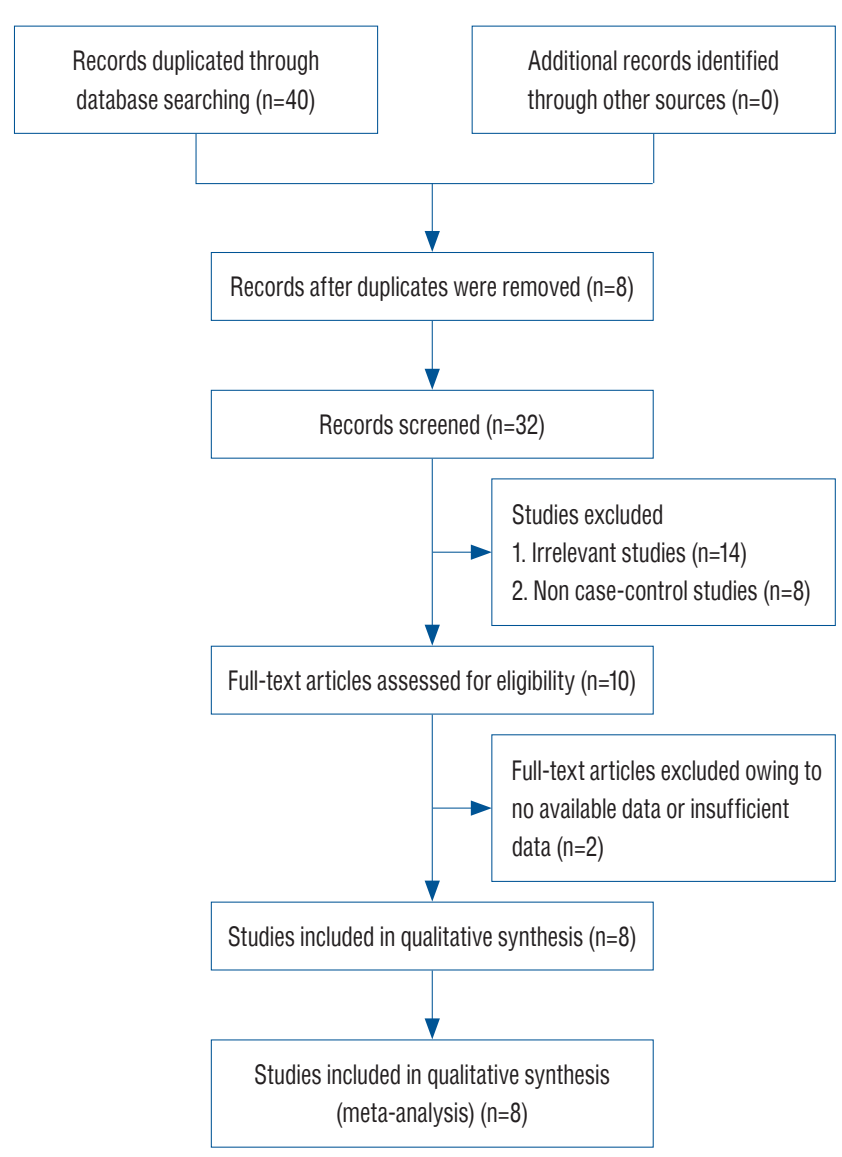

Fig. 1. Flowchart representing the article selection procedure for the meta-analysis of the correlation between E-selectin S128R polymorphism. 
ducted on the Chinese population.

Exclusion criteria : studies 1) with repeated research, already published in the literature; 2) in which the control group was not in agreement with the HWE; 3) wherein the data description was vague or essential data were not provided, and the statistical method was not suitable; 4) in which the diagnosis of IS was not in accordance with international standards; and 5) comprising reviewed literature.

\section{Methodological quality assessment}

Two reviewers, R.M. and X.T., autonomously evaluated the literature quality on the basis of the Newcastle-Ottawa Quality Assessment Scale ${ }^{11)}$. The score scale included the following aspects : 1) case group is appropriate, 1 ; 2) case group representativeness, 1 ; 3) the selection of the control group, 1 ; 4) ascertainment of the control group, 1; 5) comparability of case and control in the experimental design and statistical analysis, 2; 6) ascertainment of the exposure factor, 1; 7) identical ascertainment methods of case and control group exposure factor, 1; 8) no answer rate, 1; and 9) and those with scores
$>6$ were regarded as "high quality."

\section{Statistical analysis}

Stata version 12.0 software (Stata Corp LLC, College Station, TX, USA) was used to perform the meta-analysis based on OR value assessment with 95\% CIs to indicate the correlation between the polymorphism of E-selectin S128R and IS risk. Heterogeneity among studies was analyzed by carrying out $\mathrm{I}^{2}$ statistic and chi-square tests. If the chi-square test value was $<0.10$ and the $\mathrm{I}^{2}$ statistic surpassed $50 \%$, substantial heterogeneity was considered to exist and a random-effect model was applied to pool the data; otherwise, a fixed-effect model was applied. Publication bias was evaluated using Begg and Egger's tests. $p$ values $<0.05$ were considered to be indicative of statistical publication bias in Egger's tests. The study data were calculated with five genetic models, namely allele ( $R$ vs. S), homozygote (RR vs. SS), heterozygote (RS vs. SS), dominant (RR+RS vs. SS), and recessive models (RR vs. RS+SS).

Table 1. Characteristics of the case-control studies incorporated in the meta-analysis

\begin{tabular}{|c|c|c|c|c|c|c|c|c|c|c|c|c|}
\hline \multirow{2}{*}{ Study } & \multirow{2}{*}{ Ethnicity } & \multirow{2}{*}{$\begin{array}{l}\text { Genotyping } \\
\text { method }\end{array}$} & \multicolumn{4}{|c|}{ Cases } & \multicolumn{4}{|c|}{ Controls } & \multirow{2}{*}{$\begin{array}{c}p \text {-value for } \\
\text { HWE }\end{array}$} & \multirow{2}{*}{ Quality } \\
\hline & & & Total & RR & $\mathrm{RS}$ & SS & Total & RR & RS & SS & & \\
\hline Chen et al.') (2010) & Han & PCR-RFLP & 228 & 0 & 34 & 194 & 232 & 0 & 16 & 216 & 0.57 & 7 \\
\hline $\operatorname{Luo}^{7)}(2013)$ & Han & PCR-RFLP & 181 & 2 & 34 & 145 & 135 & 0 & 10 & 125 & 0.65 & 7 \\
\hline Tang et al. ${ }^{12)}$ (2011) & Han & PCR-RFLP & 176 & 0 & 24 & 152 & 152 & 0 & 10 & 142 & 0.67 & 6 \\
\hline Wang et al. ${ }^{13)}(2010)$ & Han & PCR-RFLP & 73 & 1 & 10 & 62 & 85 & 0 & 3 & 82 & 0.87 & 8 \\
\hline Wei et al. ${ }^{15)}(2005)$ & Zhuang & PCR-RFLP & 205 & 0 & 39 & 166 & 210 & 0 & 22 & 188 & 0.25 & 7 \\
\hline Yuan et al. ${ }^{16)}(2013)$ & Han & PCR-RFLP & 199 & 2 & 43 & 154 & 143 & 0 & 6 & 118 & 0.78 & 8 \\
\hline Zhang et al. ${ }^{19)}$ (2009) & Han & PCR-RFLP & 120 & 0 & 22 & 98 & 102 & 0 & 7 & 95 & 0.72 & 7 \\
\hline Zhao et al. ${ }^{20)}(2012)$ & Han & PCR-RFLP & 314 & 1 & 37 & 276 & 389 & 0 & 18 & 371 & 0.64 & 6 \\
\hline
\end{tabular}

HWE : Hardy-Weinberg equilibrium, PCR : polymerase chain reaction, RELP : restriction fragment length polymorphisms

Table 2. Results of the meta-analysis according to various genotype models

\begin{tabular}{|c|c|c|c|c|c|}
\hline \multicolumn{2}{|l|}{ Genetic model } & \multirow{2}{*}{ OR $(95 \% \mathrm{Cl})$} & \multirow{2}{*}{$p$-value* } & \multicolumn{2}{|c|}{ Text of heterogeneity } \\
\hline Name & Explanation & & & $p$-value, $I^{2}(\%)$ & Effect mode \\
\hline Allele model & Rvs.S & $2.75(2.15-3.51)$ & $<0.00001$ & $0.61,0$ & $\mathrm{~F}$ \\
\hline Homozygote model & RR vs. SS & $4.04(0.84-19.33)$ & 0.08 & $1.00,0$ & $\mathrm{~F}$ \\
\hline Heterozygote model & RS vs. SS & $2.50(1.95-3.19)$ & $<0.00001$ & $0.17,32$ & $\mathrm{~F}$ \\
\hline Dominant model & $R R+R S$ vs. SS & $2.85(2.21-3.67)$ & $<0.00001$ & $0.67,0$ & $\mathrm{~F}$ \\
\hline Recessive model & RR vs. RS+SS & $3.53(0.74-16.91)$ & 0.11 & $1.00,0$ & $\mathrm{~F}$ \\
\hline
\end{tabular}

${ }^{*}$ OR. OR: odds ratio, $\mathrm{Cl}$ : confidence interval, $\mathrm{F}$ : fixed-effect model 


\section{RESULTS}

\section{Data collection and characteristics of the studies}

Forty studies were selected, specifically 11 from PubMed, two from the Web of Science, four from CBM, five from CNKI, 17 from Wanfang, and one from the WIP Database. Eventually, eight studies were found to meet the criteria $^{1,7,12,13,15,16,19,20)}$, with 1478 cases and 1429 controls. All studies

\begin{tabular}{|c|c|c|c|c|c|c|}
\hline \multirow{2}{*}{ Study of subgroup } & \multicolumn{2}{|c|}{ Case } & \multicolumn{3}{|c|}{ Control } & \multirow{2}{*}{$\begin{array}{c}\text { OR } \\
\text { M.H., fixed, } 95 \% \mathrm{C}\end{array}$} \\
\hline & Event & Total & Event & Total & Weight (\%) & \\
\hline Chen et al." (2010) & 34 & 456 & 16 & 464 & 17.2 & $2.26(1.23-4.15)$ \\
\hline $\operatorname{Luo}^{7)}(2013)$ & 38 & 362 & 10 & 270 & 12.0 & $3.05(1.49-6.24)$ \\
\hline Tang et al. ${ }^{12)}(2011)$ & 24 & 352 & 10 & 304 & 11.7 & $2.15(1.01-4.57)$ \\
\hline Wang et al. ${ }^{13)}(2010)$ & 12 & 146 & 3 & 170 & 3.0 & $4.99(1.38-18.03)$ \\
\hline Wei et al. ${ }^{15)}(2005)$ & 39 & 410 & 22 & 440 & 22.6 & $2.00(1.16-3.43)$ \\
\hline Yuan et al. ${ }^{16)}(2013)$ & 4 & 398 & 6 & 248 & 7.7 & $5.40(2.27-12.83)$ \\
\hline Zhang et al. ${ }^{19)}(2009)$ & 22 & 240 & 7 & 204 & 8.1 & $2.84(1.19-6.79)$ \\
\hline Zhao et al. ${ }^{20)}(2012)$ & 39 & 628 & 18 & 778 & 17.7 & $2.80(1.58-4.94)$ \\
\hline Total $(95 \%$ Cl) & & 2992 & & 2878 & 100.0 & $2.75(2.15-3.51)$ \\
\hline Total events & 255 & & 92 & & & \\
\hline
\end{tabular}

Heterogeneity : chi $^{2}=5.40, \mathrm{df}=7(p=0.61) ; \mathrm{l}^{2}=0 \%$

Test for overall effect : $Z=8.03(p<0.00001)$

(A)

\begin{tabular}{|c|c|c|c|c|c|c|}
\hline \multirow{2}{*}{ Study of subgroup } & \multicolumn{2}{|c|}{ Case } & \multicolumn{3}{|c|}{ Control } & \multirow{2}{*}{$\begin{array}{c}\text { OR } \\
\text { M.H., fixed, } 95 \% \text { C }\end{array}$} \\
\hline & Event & Total & Event & Total & Weight (\%) & \\
\hline Chen et al." (2010) & 0 & 194 & 0 & 216 & & Not estimable \\
\hline $\operatorname{Luo}^{7)}(2013)$ & 2 & 147 & 0 & 125 & 27.4 & $4.31(0.21-90.68)$ \\
\hline Tang et a ${ }^{12)}(2011)$ & 0 & 152 & 0 & 142 & & Not estimable \\
\hline Wang et al. ${ }^{13)}(2010)$ & 1 & 63 & 0 & 82 & 21.9 & $3.96(0.16-98.86$ \\
\hline Wei et al. ${ }^{15)}(2005)$ & 0 & 166 & 0 & 188 & & Not estimable \\
\hline Yuan et al. ${ }^{16)}(2013)$ & 2 & 156 & 0 & 118 & 28.8 & $3.83(0.18-80.64)$ \\
\hline Zhang et al. ${ }^{19)}(2009)$ & 0 & 98 & 0 & 98 & & Not estimable \\
\hline Zhao et al. ${ }^{20)}(2012)$ & 1 & 277 & 0 & 371 & 21.9 & $4.03(0.16-99.3$ \\
\hline Total (95\% Cl) & & 1253 & & 1337 & 100.0 & $4.04(0.84-19$. \\
\hline Total events & 6 & & 0 & & & \\
\hline
\end{tabular}

Heterogeneity : chi $^{2}=0.00, \mathrm{df}=3(p=1.00) ;\left.\right|^{2}=0 \%$

Test for overall effect : $Z=1.75(p=0.08)$

(B)

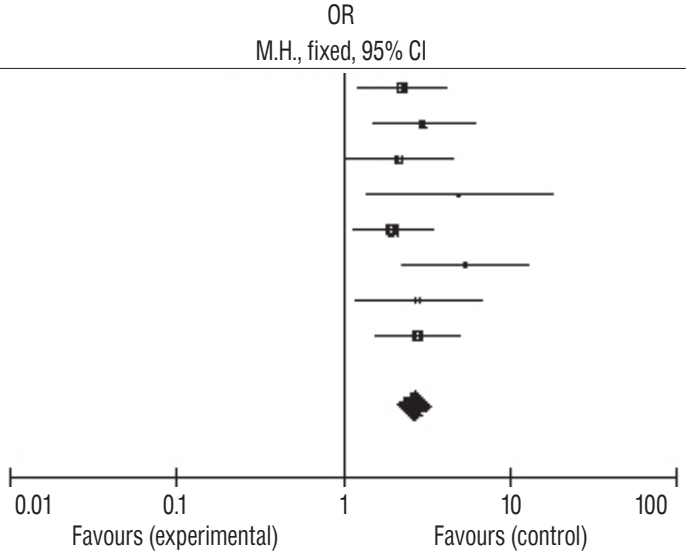

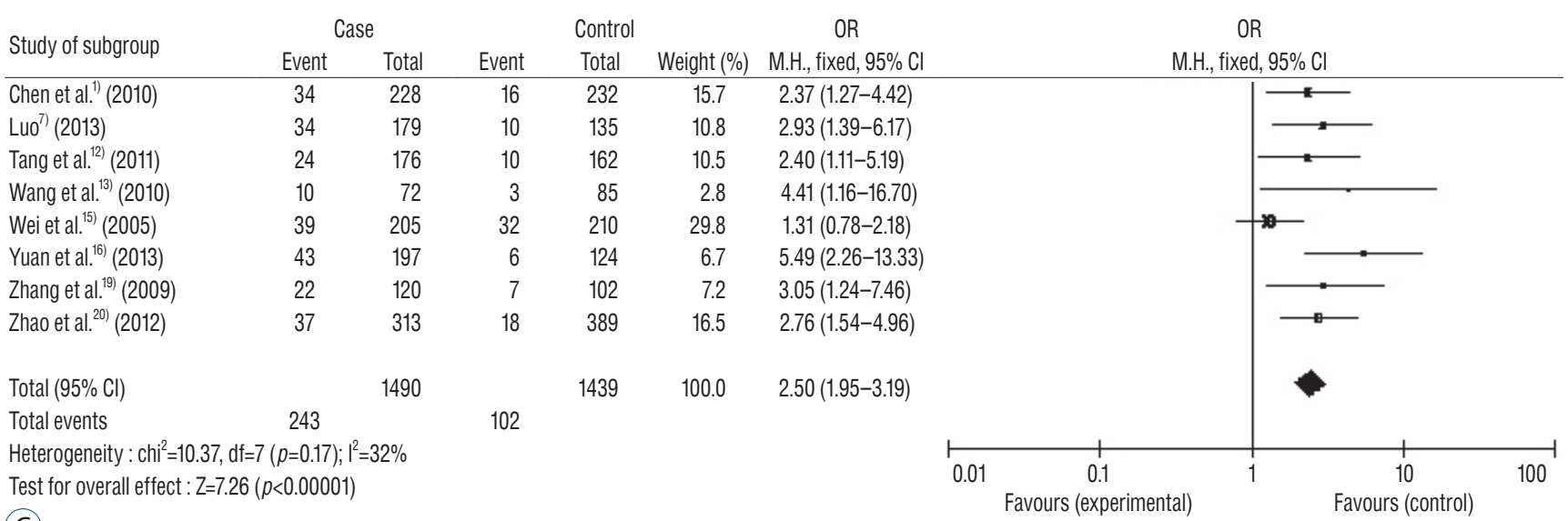

(C)

Fig. 2. Forest plot of IS risk associated with E-selectin S128R polymorphism. A : Allelic contrast model (R vs. S). B : Homozygote model (RR vs. SS). C : Heterozygote model (RS vs. SS). D : Dominant model (RR+RS vs. SS). E : Recessive model (RR vs. RS+SS). OR : odds ratio, M.H. : Mantel-Haenszel, Cl : confidence interval, IS : ischemic stroke. 


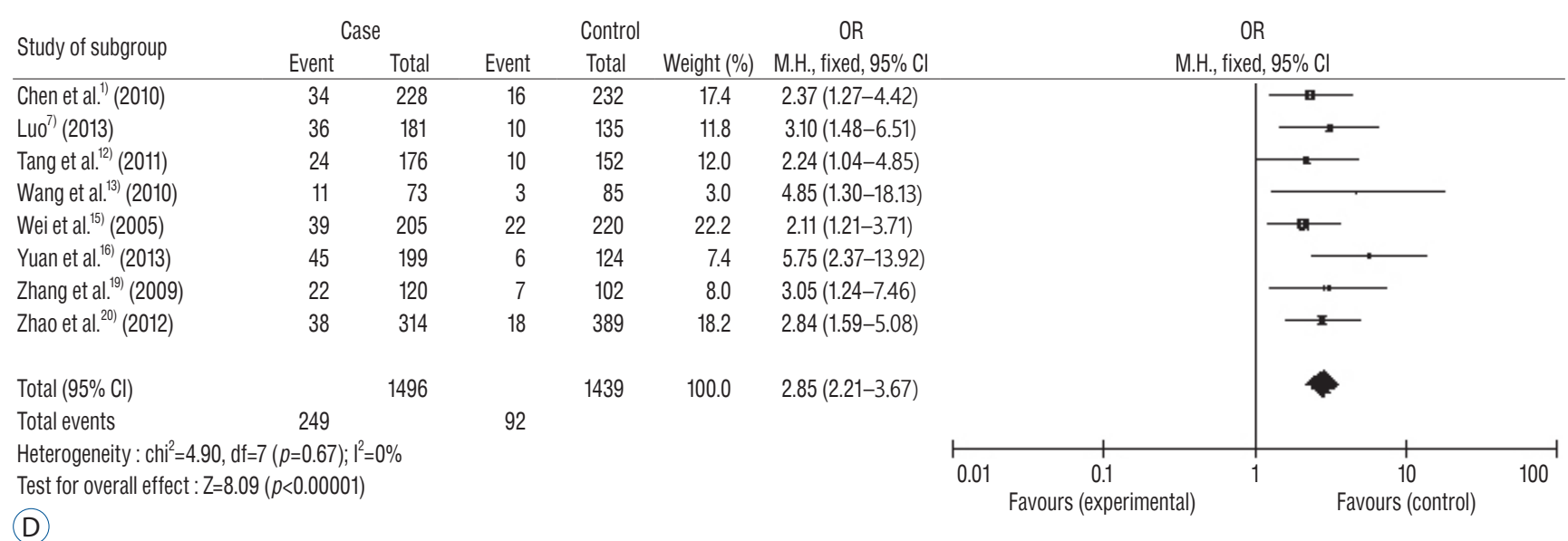

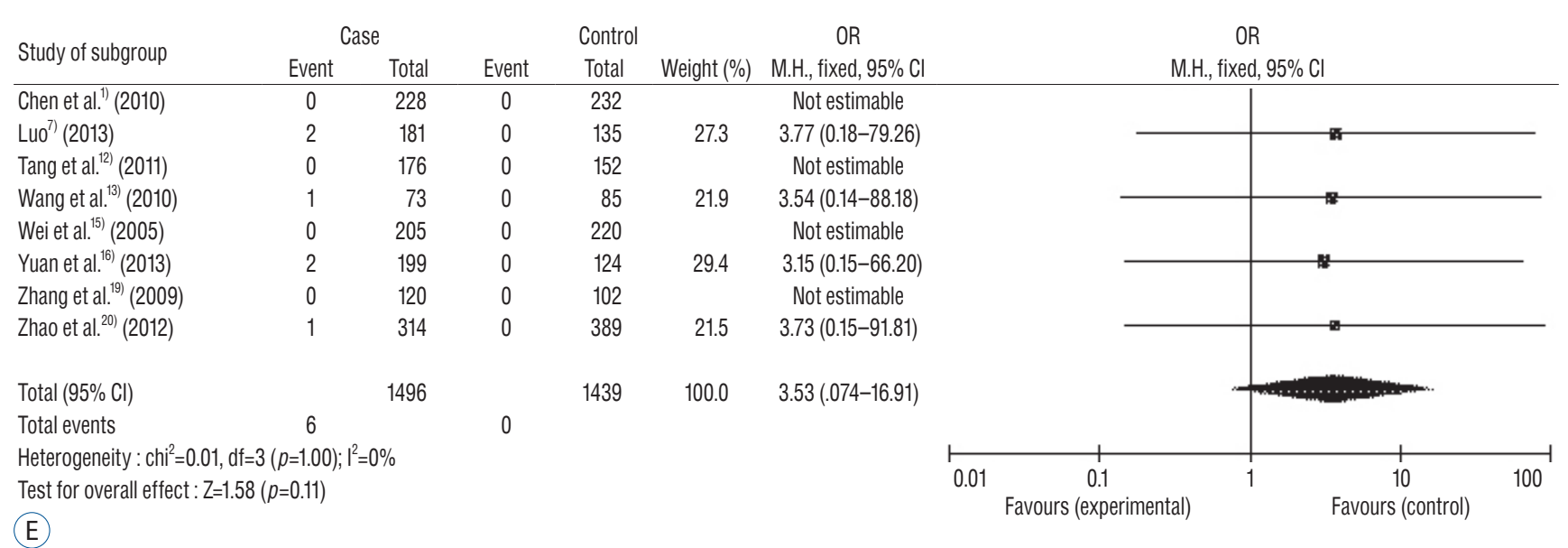

Fig. 2. Continued.

were carried out on the Chinese population, seven on the Han ethnic group, and one on the Zhuang ethnic group. The detailed procedure for the selection of the studies is presented in Fig. 1. The study characteristics including the basic literaturerelated information, the original data, the $p$ value for HWE, the genotype data, and quality assessment are shown in Table 1.

\section{Meta-analysis results}

The eight eligible case-control studies included 1478 cases and 1429 controls. The findings of this meta-analysis such as heterogeneity tests, effect models, pooled ORs with 95\% CI, and the respective $p$ values are given in Table 2. Overall, a significantly increased risk was observed with the five genetic models : allelic contrast model R vs. S : OR, 2.75; 95\% CI, 2.15-3.51; $p<0.00001$ (Fig. 2A); homozygote model RR vs. SS : OR, 4.04, 95\% CI, 0.84-19.33; $p=0.08$ (Fig. 2B); heterozygote model RS vs. SS : OR, 2.50; 95\% CI, 1.95-3.19; $p<0.00001$ (Fig. 2C); dominant model RR+RS vs. SS : OR, 2.85; 95\% CI, 2.21-
3.67; $p<0.00001$ (Fig. 2D); and recessive model RR vs. RS+SS : OR, 3.53; 95\% CI, 0.74-16.91; $p=0.11$ (Fig. 2E). A significant association between the allelic contrast, heterozygote, and dominant models and the Chinese IS population was observed.

\section{Sensitivity analysis and publication bias}

The meta-analysis publication bias was determined using funnel plots (Fig. 3), which showed a bit of publication bias in the allelic contrast and dominant gene models that might have influenced the results. Sensitivity analysis showed that deleting every single study with pooled ORs did not affect the stability of the results (Fig. 4).

\section{DISCUSSION}

The IS risk factors include a series of diseases such as ath- 

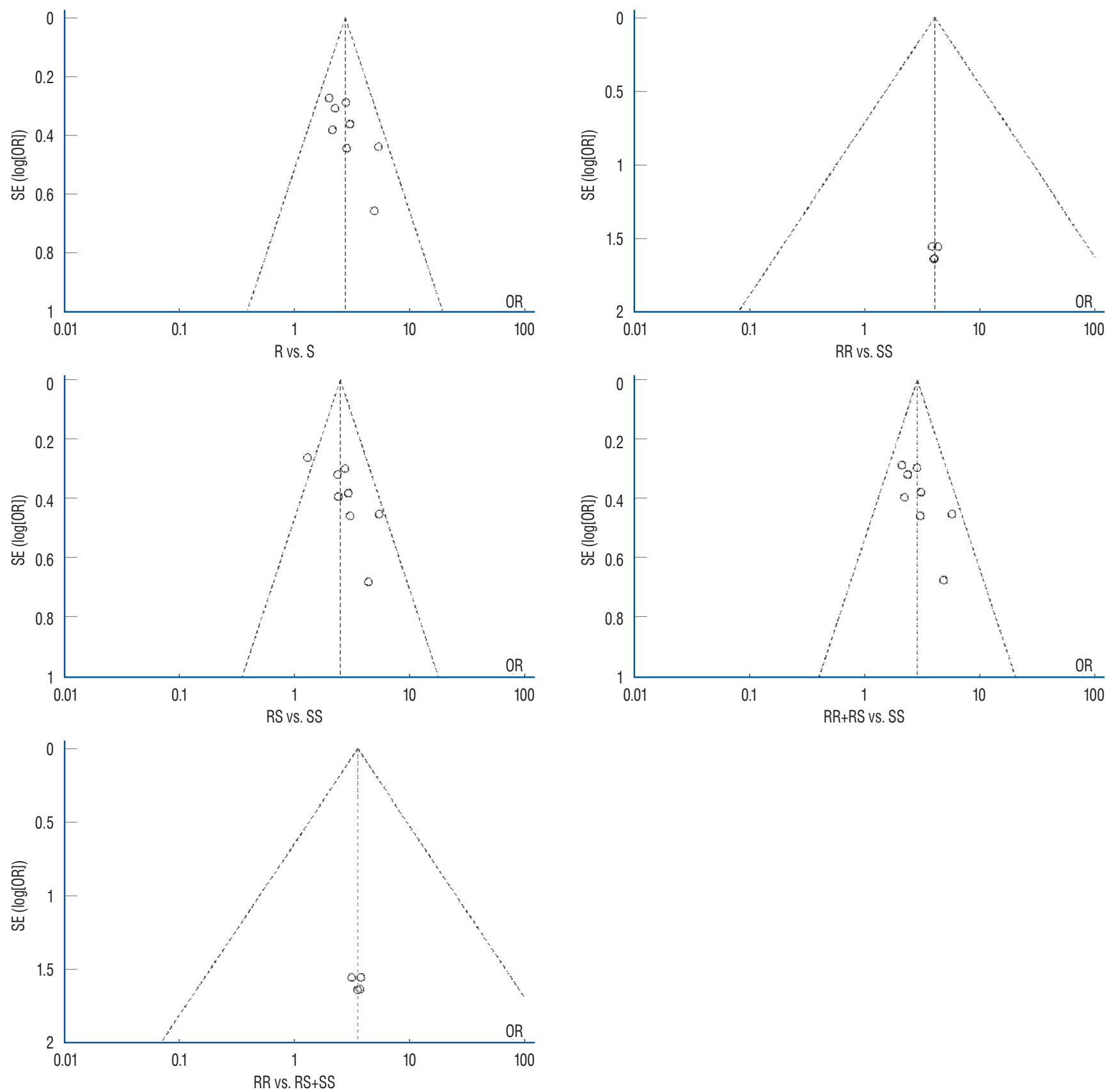

Fig. 3. Funnel plot for risk of IS associated with E-selectin S128R polymorphism. SE : standard error, OR : odds ratio; IS : ischemic stroke.

erosclerosis, dyslipidemia, hypertension, and hyperhomocysteinemia. Clinical studies have shown that not all strokes are related to diseases, and genetic factors are now being considered to play an important role in $\mathrm{IS}^{3)}$. E-selectin is one of the main members of the adhesive molecular selectin family that acts as a cell surface glycoprotein. It has a molecular weight of $115 \times 10^{3}$ with 589 amino acid residues and is only expressed on the surface of endothelial cells activated by cytokines. E-selectin is a pro-inflammatory cytokine in vivo and participates in the adhesion and aggregation of various white blood cells (including neutrophils, macrophages, monocytes, etc.). It is simultaneously involved in lipid metabolism, affecting the cholesterol, low-density lipoprotein, and high-density lipoprotein levels. The selectins are a family of carbohydrate-binding pro- 


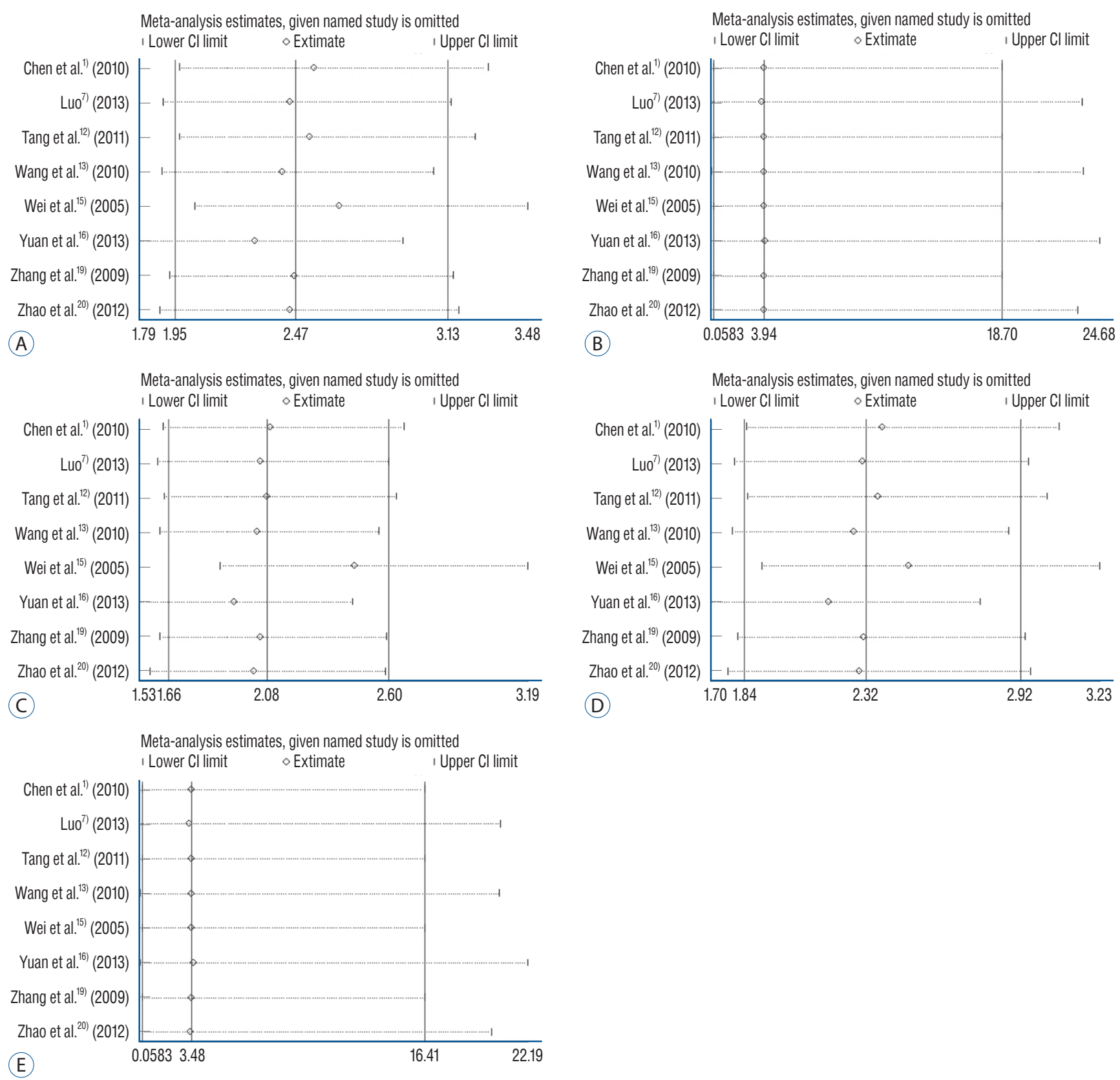

Fig. 4. Sensitivity analysis of OR summary on correlation between polymorphism of E-selectin S128Rand IS risk. A : Allelic contrast model (R vs. S). B : Homozygote model (RR vs. SS). C : Heterozygote model (RS vs. SS). D : Dominant model (RR+RS vs. SS). E : Recessive model (RR vs. RS+SS). OR : odds ratio, IS : ischemic stroke.

teins (lectins) that are differentially expressed by leukocytes and endothelial cells, which promote the accumulation of leukocytes around the areas of injury or inflammation around arterioles. E-selectin has a significant role in atherosclerosis, inflammation, and thrombosis stroke as well as other pathological processes.

It has been demonstrated that E-selectin is upregulated after focal cerebral ischemia through various animal models ${ }^{14,17)}$. At present, only a fragment of the single nucleotide polymorphisms in the E-selectin gene has been identified. The most common polymorphism of the E-selectin gene, S128R, occurs on exon 4 of the gene. Specifically, serine (Ser) substitutes the arginine (Arg) surrounding the extracellular region of the receptor, increasing the affinity for ligands and serving as a 
functional polymorphism. E-selectin S128R polymorphism is known to participate in IS to a certain extent. Therefore, this study aimed to ascertain the correlation between polymorphism of the E-selectin gene S128R and the genetic susceptibility to IS, which has a significantly immense role to play in the effective diagnosis and treatment of IS.

This meta-analysis used eight studies to review and analyze the relationship between the polymorphism of E-selection and IS among the Chinese population, although the study population contained different ethnic groups. Like most studies, our meta-analysis also showed that the $\mathrm{R}$ allele significantly increases the IS risk, indicating a positive correlation between Eselectin and IS. Although the ethnic groups in this study were different, seven studies included only Han Chinese and one focused on the Zhuang community. However, the results showed that the R allele significantly increases the risk of IS in the Han and Zhuang populations in China. The majority of the Chinese population is Han. Our meta-analysis results were as follows : R vs. S, $p<0.00001$; RS vs. SS, $p<0.00001$; and $\mathrm{RR}+\mathrm{RS}$ vs. $\mathrm{SS}, p<0.00001$. All three gene models had statistical significance. Therefore, it can be considered that the $\mathrm{R}$ allele of E-selectin and the RS genotype can significantly increase IS risk, which indicates a genetic susceptibility factor to stroke among the Chinese population. This may be attributed to cerebral vascular endothelial damage under the inflammatory condition, causing the expression of E-selectin to increase rapidly and leading to a significantly higher level of E-selectin than before. Wei et al.'s research ${ }^{15)}$ showed the polymorphism of the E-selectin gene S128R can affect the serum E-selectin level. The serum E-selectin levels of patients with the RS genotype were significantly higher than those of noncarriers. In pathological conditions, the population with the $\mathrm{R}$ allele was found to have a significantly higher IS risk, which may be attributed to the $\mathrm{R}$ allele affecting the expression of E-selectin in endothelial cells. Transformation of the genetic structure causes changes in protein function, thus promoting high expression of E-selectin and causing the IS incident to increase significantly. This is consistent with the conclusion by Mlekusch et al. ${ }^{8)}$ that the E-selectin genotype tends to influence the serum E-selectin level.

Our study had the following limitations : 1) all case-control studies were conducted mostly on the Han population and only one was conducted on the Zhuang population, thus leading to a lack of data on other populations. 2) All case-control studies had only a few and sometimes no populations with the RR genotype, which may influence the final results. 3) Only published articles were included. And 4) not all the case-control studies have intact background data for the study such as age, smoking, alcohol consumption, and other lifestyle factors. The correlational study conducted between E-selectin S128R polymorphism and IS susceptibility among the Chinese population can be implemented as case-control, multicenter studies on larger samples in various nations.

\section{CONCLUSION}

To conclude, this study indicated that the E-selectin R allele and RS genotype may be risk factors for IS. Further investigations with a larger sample size and detailed gene and environmental data are needed to confirm the findings of this study.

\section{CONFLICTS OF INTEREST}

No potential conflict of interest relevant to this article was reported.

\section{INFORMED CONSENT}

This type of study does not require informed consent.

\section{AUTHOR CONTRIBUTIONS}

\author{
Conceptualization : $\mathrm{XY}$ \\ Data curation : XY \\ Formal analysis : RM \\ Funding acquisition : GW, XY \\ Methodology: YZ \\ Project administration : GW \\ Visualization : $\mathrm{YZ}$ \\ Writing - original draft $: \mathrm{XY}$ \\ Writing - review \& editing : GW
}




\section{ORCID}

Xitong Yang https:/orcid.org/0000-0002-6203-3313

Rong Ma https://orcid.org/0000-0002-1866-955X

Yuanyuan Zhang https://orcid.org/0000-0002-5263-4895

Guangming Wang https://orcid.org/0000-0002-0220-1493

\section{- Acknowledgements}

This work was supported by the National Natural Science Foundation of China (No. 81360206), Reserve Talents of Academic and Technical Leaders of Young and Middle-aged people in Yunnan Province (No. 2014HB025), Yunnan Medical Science Leader (No. D-2017057), and Yunnan Science and Technology Project (2017DQC02).

\section{References}

1. Chen JN, Wei GY, Fu XL, Li ZX, Liang LP, Deng YY, et al. : Relationship of E-selectin A561C gene polymorphisms in patients with cerebral infarction and its effect on plasma lipid level. CHN J of Mod Medicine 20 : 2468-2470, 2010

2. Das S, Roy S, Kaul S, Jyothy A, Munshi A : E-selectin gene (S128R) polymorphism in hemorrhagic stroke: comparison with ischemic stroke. Neurosci Lett 581 : 125-128, 2014

3. Haidari M, Hajilooi M, Rafiei AR, Rezaii AA, Hoseinipanah SM : Eselectin genetic variation as a susceptibility factor for ischemic stroke. Cerebrovasc Dis 28 : 26-32, 2009

4. Katan M, Luft A : Global burden of stroke. Semin Neurol 38 : 208211, 2018

5. Kumar A, Misra S, Sagar R, Kumar P, Yadav AK, Talwar P, et al. : Relationship between factor $V$ Leiden gene variant and risk of ischemic stroke: a case-control study. Ann Indian Acad Neurol 20 : 284-288, 2017

6. Losey P, Ladds E, Laprais M, Geuvel B, Burns L, Bordet R, et al. : The role of PPAR activation during the systemic response to brain injury. J Neuroinflammation $12: 1-10,2015$
7. Luo D : Association study between a polymorphism of E-selectin S128R gene and stroke. CHN J of Med Inform 26 : 326-327, 2013

8. Mlekusch W, Exner M, Schillinger M, Sabeti S, Mannhalter C, Minar E, et al. : E-Selectin and restenosis after femoropopliteal angioplasty: prognostic impact of the Ser128Arg genotype and plasma levels. Thromb Haemost 91 : 171-179, 2004

9. Roy S, Das S, Danaboina R, Sharma V, Kaul S, Jyothy A, et al. : Association of E-selectin gene polymorphism (S128R) with ischemic stroke and stroke subtypes. Inflammation 37 : 599-603, 2014

10. Srivastava K, Chandra S, Narang R, Bhatia J, Saluja D : E-selectin gene inessential hypertension: a case-control study. Eur J Clin Invest 48 : e12868, 2018

11. Stang A : Critical evaluation of the Newcastle-Ottawa scale for the assessment of the quality of nonrandomized studies in meta-analyses. Eur J Epidemiol 25 : 603-605, 2010

12. Tang $\mathrm{TT}$, Ren $\mathrm{TL}$, Zhang $\mathrm{YL}$ : Analysis of $\mathrm{A} 561 \mathrm{C}$ gene polymorphism in exon 4 of E- selectin in 176 stroke patients. SD Medical J 51 : 77-78, 2011

13. Wang F, Liu RZ, Liu ZW : Association of Plasma E-selectin, S128R Gene Polymorphisms and Cerebral Infarction in Shanxi Han Population. CHN J of Integ Med on Cardio/Cerebrovascular Dis 8 : 549-551, 2010

14. Wang $X$, Feuerstein GZ : Induced expression of adhesion molecules following focal brain ischemia. J Neurotrauma 12 : 825-883, 1995

15. Wei YS, Qunqing XU, Meng L, Liang F : Polymorphisms of E-selectin gene and genetic susceptibility of ischemic stroke in Zhuang nationality of Guangxi province. J Third Mil Med Univ 27 : 2160-2163, 2005

16. Yuan D, Kan CY, Gao P : Association study between a polymorphism of E-selectin gene and cerebral infarction. J of Apoplexy and Nerv Dis $30: 208-210,2013$

17. Zhang R, Chopp $M$, Zhang Z, Jiang N, Powers $C$ : The expression of $\mathrm{P}$ - and $\mathrm{E}$-selectins in three models of middle cerebral artery occlusion. Brain Res 785 : 207-214, 1998

18. Zhang Y, Wang $X, X u X$, Chen $R$, Kan $H$ : Stock volatility and stroke mortality in a Chinese population. J Cardiovasc Med (Hagerstown) 14 : 617-621, 2013

19. Zhang YL, Tang T, Fang B : Polymorphisms of E-selectin S128R gene and genetic susceptibility of cerebral thrombosis. CHN J of Med Inform 19 : 1989-1991, 2009

20. Zhao DX, Feng J, Cong SY, Zhang W : Association of E-selectin gene polymorphisms with ischemic stroke in a Chinese Han population. J Neurosci Res 90 : 1782-1787, 2012 